\title{
EFEITO DOS COMPOSTOS NATURAIS BIOATIVOS NA CONSERVAÇÃO PÓS-COLHEITA DE FRUTOS DE MANGUEIRA CV. TOMMY ATKINSS
}

\author{
Effects of bioactive natural compounds on the postharvest conservation \\ of mango fruits cv. Tommy Atkins
}

\author{
Maria Julia da Silva Cruz', Edmar Clemente' ${ }^{2}$, Maria Eugênia da Silva Cruz², \\ Freddy Mora ${ }^{3}$, Leocir Cossaro ${ }^{4}$, Noemi Pelisson ${ }^{4}$
}

\begin{abstract}
RESUMO
A antracnose causa severos danos na pós-colheita de manga. Como o controle químico tem demonstrado desvantagens, o potencial uso de óleos essenciais para o controle de fitopatógenos tem sido uma alternativa. Neste trabalho, objetvou-se avaliar a eficácia de produtos alternativos no controle da antracnose e na conservação de mangas (Mangifera indica) cv. Tommy Atkins tratado com bicarbonato de sódio diluído em água destilada, na concentração de 3\% (v/v), via imersão. Como tratamento, foram utilizados os óleos essenciais de Origanum majorana, Citrus sinensis, Cymbopogon citratus e Eucalyptus citriodora, via fumigação, na dosagem de $1000 \mu \mathrm{L}$ e água destilada via fumigação; todos por 3 minutos. Frutos da Unidade CEASA-Maringá/PR, selecionados, pesados em balança semianalítica, desinfetados em imersão por 3 minutos em solução de hipoclorito de sódio a $0,5 \%$ (v/v); foram submetidos aos tratamentos, permanecendo sob condições ambientes $\left(26 \pm 2{ }^{\circ} \mathrm{C}\right.$ e UR $\left.90 \pm 5 \%\right)$. Exceto o tratamento com Citrus sinensis, que proporcionou um $\mathrm{pH}$ maior do que a testemunha, diferindo significativamente dos demais tratamentos que elevaram a variável. A redução da acidez em todos os tratamentos e aumentos dos sólidos solúveis totais nos frutos, sendo que o tratamento com bicarbonato de sódio proporcionou $14,42^{\circ}$ Brix. O número de frutos com antracnose foi menor no tratamento com Citrus sinensis e este diferiu dos demais comprovando o efeito de espécies do gênero Citrus como antifúngicos. O uso de óleos essenciais como o de Citrus sinensis controlou a antracnose e manteve a qualidade dos frutos.
\end{abstract}

Termos para indexação: Mangifera indica, pós-colheita, óleo essencial, antracnose.

\begin{abstract}
The anthracnose causes severe damages to the mango post-harvest. As chemical control has shown disadvantages, the potential of use of essential oils has been an alternative for the phytopathogen control. The objective of the present work was to evaluate the efficacy of alternative products in the anthracnose control and in the conservation of mangos (Mangifera mandica) cv. Tommy Atkins treated with sodium bicarbonate diluted in distilled water, concentration of $3 \%(\mathrm{v} / \mathrm{v})$, through immersion. For the treatment, the essential oils of Origanum majorana, Citrus sinensis, Cymbopogon citratus and Eucalyptus citriodora were used through fumigation, in the dosage of $1000 \mu \mathrm{L}$ and distilled water through fumigation; all for 3 minutes. Mango fruits of the Unity CEASA-Maringá/PR, selected, weighed in a semi-analytical scale, disinfected by immersion of 3 minutes in a solution of sodium hypochlorite to $0.5 \%(\mathrm{v} / \mathrm{v})$; rinsed in running water and dried. They were submitted to the treatments under ambient conditions $(26$ $\pm 2^{\circ} \mathrm{C}$ and $\mathrm{RH} 90 \pm 5 \%$ ). Except for the treatment of Citrus sinensis, that resulted in a pH larger than the witness, significantly differing from the treatments that elevate the variable. There was reduction of the acidity in all of the treatments and increment of TSS in the fruits and with sodium bicarbonate provided $14.42^{\circ}$ Brix. The number of fruits with anthracnose was smaller in those treated with Citrus sinensis and this differed from the others, proving the effect of Citrus as fungitoxic. The use of essential oils such as that obtained from Citrus sinensis controlled the anthracnose and maintained the quality of the fruits.
\end{abstract}

Index terms: Mangifera indica, postharvest, essential oil, anthracnose.

(Recebido em 5 de março de 2007 e aprovado em 15 de dezembro de 2008)

\section{INTRODUÇÃO}

O Brasil, mesmo estando entre os maiores produtores mundiais de frutas, ocupando a segunda posição em volume produzido, se coloca-se em desvantagem quando se verifica o volume de perdas ocorridas entre a colheita e o consumo. Grande parte desse total não chega à mesa do consumidor, pois, estima-se que 10 milhões de toneladas são perdidos por ano, correspondendo a 30-40\% da produção (Benato et al., 2001; Jacomino, 2003). A manga é produzida em grande quantidade durante um período curto de tempo e deteriorase rapidamente (Soares Junior, 2003). Estima-se que, no Brasil, as perdas pós-colheita de manga oscilem entre $40 \%$ e 50\%, principalmente por manuseio e preservação inadequados (Leite et al., 1998).

\footnotetext{
1Universidade Estadual de Maringá/UEM - Departamento de Agronomia/DGA - Maringá, PR

2Universidade Estadual de Maringá/UEM - Departamento de Química/DQI - Maringá, PR - eclemente@uem.br

3Universidad de Concepción/UDEC - Faculdad de Ciencias Florestales - Departamento de Silvicultura - Concepción, Chile

${ }^{4}$ Universidade Estadual de Maringá/UEM - Departamento de Agronomia - Maringá, PR
} 
A produção de manga voltada para o mercado de produto de qualidade exigiu cada vez mais, novas tecnologias, mão-de-obra qualificada e serviços especializados, tanto no processo produtivo, quanto nas atividades pós-colheita. Por tratar-se de um produto que se consome principalmente 'in natura', tornam-se importantes às limitações relativas à conservação, embalagem e transporte da fruta. A qualidade da fruta que chega ao mercado consumidor deve atender às exigências de aparência, firmeza, cor, odor, dentre outros.

Pesquisas em pós-colheita buscam estudar técnicas de armazenamento que prolonguem a vida útil da fruta, incluindo o controle de patógenos que constituem uma das principais causas de perdas das frutas na fase de póscolheita. O controle químico de patógenos apresenta vantagens e desvantagens, sendo estas últimas, de maior expressão, visto que alguns fungicidas, além do dano ao meio ambiente e à saúde humana, têm proporcionado o surgimento de estirpes resistentes de certos fungos, como observado por vários pesquisadores. $\mathrm{O}$ uso de plantas medicinais para o controle de fitopatógenos tem sido demonstrado em diversos trabalhos (Caccioni et al., 1995; Almada-Ruiz et al., 2003; USDA, 2003; Cruz et al., 2005a,b). Cruz et al. (2005a), por exemplo, avaliaram extratos vegetais de Azadirachta indica e cítrico, com atividade biológica antimicrobiana (2,0 e 4,0 \%) na conservação e controle de Colletotrichum musae em bananas na pós-colheita, verificaram que a redução do peso, dos diâmetros e alteração da cor externa das bananas foi menor quando submetidos aos tratamentos com produtos vegetais, além de menor incidência de antracnose. Cruz et al. (2005b) também estudaram soluções de extrato cítrico e de Azadirachta indica a 0,5 e 1,0\% na conservação de maçãs e no controle de Penicillium expansum e verificaram que os extratos foram eficazes na conservação dos frutos e no controle do patógeno, sendo que todos os tratamentos levaram a comprovação potencial dos compostos secundários de plantas. Considerando que produtos perecíveis são organismos vivos que, pós - colheita, têm continuidade de seu processo vital normal, com várias restrições aos métodos convencionais de controle de patógenos, conduziu-se este trabalho, com o objetivo de avaliar a eficácia de produtos alternativos no controle de antracnose e na conservação de manga na pós-colheita.

\section{MATERIAL E MÉTODOS}

Frutos de mangueira "Tommy Atkins", provenientes da Unidade CEASA-Maringá/PR, estádio 4, devidamente selecionados e pesados em balança semianalítica, foram desinfetados superficialmente, por meio da imersão, por 3 minutos, em solução de hipoclorito de sódio a $0,5 \%$ (v/v) e enxaguado em água corrente. Após a secagem, os frutos foram submetidos aos tratamentos com bicarbonato de sódio diluído em água destilada, na concentração de $3 \%$ (v/v) por 3 minutos, via imersão; óleos essenciais de Origanum majorana, Citrus sinensis, Cymbopogon citratus e Eucalyptus citriodora, via fumigação, na dosagem de $1000 \mu \mathrm{L}$ /por 3 minutos e água destilada por 3 minutos, via fumigação. Cada tratamento foi composto por quatro repetições de 8 frutos, sendo que o tratamento testemunha constituiu-se por frutos tratados com água destilada, aplicados na forma de imersão. Os frutos submetidos aos tratamentos permaneceram sob condições ambientes $\left(26 \pm 2{ }^{\circ} \mathrm{C}\right.$ e UR $\left.90 \pm 5 \%\right)$, acondicionados em bandejas plásticas e envoltos por sacos de polietileno. A cada sete dias, durante 21 , dias os frutos foram avaliados quanto às seguintes variáveis: (a) Sólidos solúveis totais (SST): por leitura em refratômetro (AOAC, 1992). (b) Acidez total titulável (ATT): pela titulação de 10 $\mathrm{mL}$ de suco diluído em $100 \mathrm{~mL}$ de água destilada, com uma solução de $\mathrm{NaOH}$ 100mmoL; (c) pH: determinado em potenciômetro (Instituto Adolfo Lutz, 1985) e (d) incidência de antracnose: calculada a partir do número de frutos infectados pelo patógeno. Os dados de pH, ATT e SST foram submetidos à análise de variância, aplicando-se o teste de Scott-Knott a 5\%, e nos casos de interação significativa, foram realizadas análises de regressão, a partir do desdobramento dos tratamentos com o programa estatístico SISVAR (Ferreira, 2000). Considerou-se o número de frutos doentes com antracnose medida num período total de 21 dias, em um delineamento inteiramente ao acaso com os tratamentos como única fonte de variação, onde se utilizou Modelos Lineares Generalizados considerando-se distribuição de Poisson com função de ligação logarítmica (canônica) (Myers et al., 2002).

\section{RESULTADOS E DISCUSSÃO}

Avaliando o potencial hidrogeniônico na Tabela 1, verifica-se que, com o decorrer do tempo de armazenamento e avanço da maturação, ocorreu elevação do pH em todos os frutos submetidos aos diferentes tratamentos, sendo que nos frutos que constituíram a testemunha tiveram menor alteração. No $1^{\circ}$ dia, aqueles frutos encontravam-se com pH de 4,06 e ,no $21^{\circ}$ dia, o valor foi de 4,18. Nos demais, foram maiores as variações no $\mathrm{pH}$ com o decorrer do tempo, sendo que o tratamento com $\mathrm{H}_{2} \mathrm{O}$ fumigação, conduziu a maior variação do $\mathrm{pH}\left(4,17\right.$ no $1^{\circ}$ dia e 4,84 no $21^{\circ}$ dia). Verificou-se diferenças significativas entre os tratamentos, originando desdobramento do tratamento dentro de cada nível de tempo de armazenamento para a variável $\mathrm{pH}$. 
Tabela 1 - Valores médios de $\mathrm{pH}$ de frutos de mangueira "Tommy Atkins", submetidos aos distintos tratamentos em função do tempo de armazenamento (TA) sob condições ambientais.

\begin{tabular}{lllll}
\hline Tratamentos/TA & $1^{\circ}$ dia & $7^{\circ}$ dia & $14^{\circ}$ dia & $21^{\circ}$ dia \\
\hline Testemunha & $4,06 \mathrm{c}$ & $4,11 \mathrm{c}$ & $4,14 \mathrm{~d}$ & $4,18 \mathrm{e}$ \\
$\mathrm{H}_{2} \mathrm{O}$ fumigação & $4,17 \mathrm{a}$ & $4,24 \mathrm{a}$ & $4,43 \mathrm{a}$ & $4,84 \mathrm{a}$ \\
Bicarbonato de sódio & $4,11 \mathrm{~b}$ & $4,22 \mathrm{a}$ & $4,36 \mathrm{~b}$ & $4,68 \mathrm{~b}$ \\
Origanum majorana & $4,03 \mathrm{c}$ & $4,06 \mathrm{~d}$ & $4,21 \mathrm{c}$ & $4,42 \mathrm{~d}$ \\
Citrus sinensis & $4,09 \mathrm{~b}$ & $4,11 \mathrm{c}$ & $4,13 \mathrm{~d}$ & $4,45 \mathrm{~d}$ \\
Cymbopogon citratus & $4,13 \mathrm{a}$ & $4,17 \mathrm{~b}$ & $4,37 \mathrm{~b}$ & $4,63 \mathrm{c}$ \\
Eucalyptus citriodora & $4,09 \mathrm{a}$ & $4,16 \mathrm{~b}$ & $4,24 \mathrm{c}$ & $4,59 \mathrm{c}$ \\
\hline
\end{tabular}

Médias seguidas por letras iguais na coluna não diferem entre si, pelo teste de Skott-Knott ao nível de 5\%.

A manga é uma fruta cuja maioria dos cultivares apresenta valores de $\mathrm{pH}$ abaixo de 4,5 (Berniz, 1984). Carvalho et al. (2004) encontraram valores de $\mathrm{pH}$ para a cultivar Tommy Atkins de 4,37 + 0,64, enquanto que Martim (2006) encontrou valor de 4,41 $\pm 0,10$ nas frutas da mesma cultivar. Tais resultados estão em concordância com os resultados obtidos neste trabalho; sendo uma fruta climatérica, com a evolução da maturação, há o consumo de ácidos orgânicos no processo de respiração, ocorrendo a diminuição da acidez e elevação do pH. Observa-se (Tabela 1) que, aos 7 dias, todos os tratamentos, exceto o com Citrus sinensis, diferiram significativamente da testemunha; aos 14 dias, todos os tratamentos, exceto o tratamento com $C$. sinensis, diferiram significativamente da testemunha e, aos 21, dias todos os tratamentos diferiram significativamente da testemunha, proporcionando um $\mathrm{pH}$ maior do que o encontrado nos frutos da testemunha, levando-nos a sugerir que os tratamentos empregados, comparando-se com os resultados da testemunha na presente tabela, conduziram à elevação desta variável. Ocorreu um decréscimo na acidez total titulável em todos os tratamentos (Tabela 2), ao longo do tempo de armazenamento, sendo que a maior redução na acidez total ocorreu nos frutos da testemunha. Aos 7 dias, suas frutas, juntamente com aquelas submetidas aos tratamentos com $\mathrm{H}_{2} \mathrm{O}$ fumigação e Citrus sinensis, não diferiram entre si, mas diferiram significativamente dos demais tratamentos; aos 14 dias os da testemunha e aqueles submetidos ao tratamento com $C$. sinensis, não diferiram entre si, mas diferiram dos demais tratamentos e, aos 21 dias, o maior valor de acidez total foi o dos tratados com $C$. sinensis. Ocorreram diferenças significativas dos tratamentos dentro de cada nível tempo de armazenamento para ATT.

A redução da A.T.T. em todos os tratamentos, está de acordo com a fisiologia de frutos climatéricos, onde os ácidos orgânicos são metabolizados e utilizados na respiração (Chitarra \& Chitarra, 2005), entretanto os valores encontrados neste trabalho são maiores que os valores encontrados por outros autores, visto que Kaneshiro et al. (1995) relatam valor de 0,5\% em polpa de mangas verdes e de $0,15 \%$ em mangas maduras "Tommy Atkins" e Lederman (1998), citam valores de 1,14 a 0,62\% para a mesma cultivar. Silva et al. (1986) determinaram para outras mangas brasileiras como a variedade 'Rosa', 'Coité', 'Jasmin', 'Espada' e 'Itamaracá', nos estádios 'de vez' e 'maduro' valores de ATT compreendidos entre 2,97 a 0, 18, também inferiores aos obtidos neste trabalho. Presume-se que a acidez total titulável determinada neste trabalho, seria continuadamente reduzida, caso o tempo de armazenagem se prolongasse (maior que 21 dias), sendo que os tratamentos com Citrus sinensis, Origanum majorana e Eucalyptus citriodora retardaram a degradação dos ácidos orgânicos, visto que os frutos destes tratamentos encontravam-se, aos 21, dias com os maiores valores, respectivamente $2,45,2,26$ e 2,21 $\mathrm{g}$ de ácido cítrico/100 mL (Tabela 2), em razão dos tratamentos.

Na Tabela 3, observa-se que ocorreu um acréscimo SST nas mangas submetidas aos distintos tratamentos com o armazenamento, sendo que no $1^{\circ} \mathrm{dia}$, as frutas do tratamento com $\mathrm{H}_{2} \mathrm{O}$ fumigação e as da testemunha não diferiram entre si, mas diferiram significativamente dos demais tratamentos; aos 7 dias de armazenamento a da testemunha encontrava-se com $8,41^{\circ}$ Brix, diferindo dos valores de todos os demais tratamentos; aos 14 dias de armazenamento, as frutas submetidas aos tratamentos com bicarbonato de sódio e Citrus sinensis, não diferiram entre si quanto aos valores de SST, mas diferiram dos demais tratamentos e, aos 21 dias, o tratamento com bicarbonato de sódio, proporcionou a obtenção do maior teor de sólidos solúveis, seguido pelas frutas submetidas ao tratamento com $C$. sinensis, sendo que diferiram dos valores obtidos nos demais tratamentos. 
Tabela 2 - Valores médios de ATT. (expressa em g de acido cítrico/100 mL) de frutos de mangueira "Tommy Atkins", submetidos aos distintos tratamentos, em função do tempo de armazenamento (TA), sob condições ambientais.

\begin{tabular}{lcccc}
\hline Tratamentos/TA & $1^{\circ}$ dia & $7^{\circ}$ dia & $14^{\circ}$ dia & $21^{\circ}$ dia \\
\hline Testemunha & $3,13 \mathrm{~b}$ & $2,87 \mathrm{a}$ & $2,74 \mathrm{a}$ & $1,75 \mathrm{~d}$ \\
$\mathrm{H}_{2} \mathrm{O}$ fumigação & $3,03 \mathrm{~b}$ & $2,84 \mathrm{a}$ & $2,03 \mathrm{~d}$ & $1,76 \mathrm{~d}$ \\
Bicarbonato de sódio & $2,84 \mathrm{c}$ & $2,61 \mathrm{~b}$ & $2,21 \mathrm{c}$ & $1,91 \mathrm{c}$ \\
Origanum majorana & $2,72 \mathrm{c}$ & $2,65 \mathrm{~b}$ & $2,47 \mathrm{~b}$ & $2,26 \mathrm{~b}$ \\
Citrus sinensis & $3,52 \mathrm{a}$ & $2,97 \mathrm{a}$ & $2,77 \mathrm{a}$ & $2,45 \mathrm{a}$ \\
Cymbopogon citratus & $2,13 \mathrm{~d}$ & $1,95 \mathrm{c}$ & $1,47 \mathrm{e}$ & $1,34 \mathrm{e}$ \\
Eucalyptus citriodora & $2,72 \mathrm{c}$ & $2,61 \mathrm{~b}$ & $2,58 \mathrm{~b}$ & $2,21 \mathrm{~b}$ \\
\hline
\end{tabular}

Médias seguidas por letras iguais na coluna não diferem entre si, pelo teste de Skott-Knott ao nível de 5\%.

Tabela 3 - Valores médios de SST ( ${ }^{\circ}$ Brix) de mangas "Tommy Atkins", submetidas aos distintos tratamentos em função do TA sob condições ambientais $\left(26 \pm 2^{\circ} \mathrm{C}\right.$ e UR $\left.90 \pm 5 \%\right)$.

\begin{tabular}{lcccc}
\hline Tratamentos & $1^{\circ}$ dia & $7^{\circ}$ dia & $14^{\circ}$ dia & $21^{\circ}$ dia \\
\hline Testemunha & $7,12 \mathrm{~b}$ & $8,41 \mathrm{~b}$ & $9,91 \mathrm{~b}$ & $11,00 \mathrm{c}$ \\
$\mathrm{H}_{2} \mathrm{O}$ fumigação & $7,55 \mathrm{~b}$ & $9,73 \mathrm{a}$ & $10,16 \mathrm{~b}$ & $11,26 \mathrm{c}$ \\
Bicarbonato de sódio & $8,30 \mathrm{a}$ & $9,73 \mathrm{a}$ & $10,77 \mathrm{a}$ & $14,42 \mathrm{a}$ \\
Origanum majorana & $8,93 \mathrm{a}$ & $9,68 \mathrm{a}$ & $10,16 \mathrm{~b}$ & $10,97 \mathrm{c}$ \\
Citrus sinensis & $9,03 \mathrm{a}$ & $10,42 \mathrm{a}$ & $10,92 \mathrm{a}$ & $11,95 \mathrm{~b}$ \\
Cymbopogon citratus & $9,10 \mathrm{a}$ & $9,46 \mathrm{a}$ & $10,02 \mathrm{~b}$ & $10,84 \mathrm{c}$ \\
Eucalyptus citriodora & $8,74 \mathrm{a}$ & $9,50 \mathrm{a}$ & $10,30 \mathrm{~b}$ & $10,96 \mathrm{c}$ \\
\hline
\end{tabular}

Médias seguidas por letras iguais na coluna não diferem entre si, pelo teste de Skott-Knott ao nível de 5\%.

O teor de SST determinado pelo refratômetro visa à obtenção de valores estimados de açúcares presentes nas frutas, se bem que pectinas, sais e ácidos também estejam inclusos nesse teor, sendo que para mangas podem variar de 6,65 a 21,9\%, dependendo do cultivar e do estádio de maturação da fruta (Awasthi \& Pandey, 1980). A cultivar Tommy Atkins apresenta valores mais baixos, variando de 7,5 a 12,0 ${ }^{\circ}$ Brix (Lima, 1997; Lederman, 1998) em frutas com idades variando de 95 a 125 dias após antese, sendo que Carvalho et al. (2004), relatam valor de $16,6 \pm 2,2^{\circ}$ Brix para esta cultivar. Podemos concluir que a hidrólise de amido, que ocorre durante o amadurecimento dos frutos esteve presente nos frutos deste trabalho, sendo o teor de SST afetado de forma significativa por todos os tratamentos avaliados e, aos 21 dias, o tratamento com bicarbonato de sódio proporcionou a obtenção do maior teor $\left(14,42^{\circ}\right.$ Brix $)$, que, provavelmente, aumentaria se o tempo de armazenamento fosse prolongado.

Na Tabela 4, observa-se que o número de frutos com antracnose foi menor no tratamento com Citrus sinensis, sendo que este tratamento diferiu dos demais. A eficácia de produtos oriundos de Citrus como antifúngico também foi verificada por outros autores. Houve diferença significativa para a fonte de variação, decorrente do tratamento (Qui-quadrado=21,17; -valor= 0,0017). Cruz (2003) verificou que produtos oriundos de espécies do gênero Citrus apresentam potencial para a utilização como antifúngicos em tratamentos pós-colheita, visando ao controle de bolor verde em laranjas, considerando-se o controle obtido ter sido superior a $94 \%$.

Tabela 4 - Valores médios da incidência de antracnose (expressa em número de frutos doentes) em frutos de mangueira "Tommy Atkins", submetidos aos distintos tratamentos.

\begin{tabular}{lc}
\hline Tratamentos & Média $\left(\mathrm{n}^{\circ}\right.$ de frutos $)$ \\
\hline Testemunha & $2,50 \mathrm{ab}$ \\
$\mathrm{H}_{2} \mathrm{O}$ fumigação & $2,25 \mathrm{~b}$ \\
Bicarbonato de sódio & $2,88 \mathrm{ab}$ \\
Origanum majorana & $2,38 \mathrm{ab}$ \\
Citrus sinensis & $0,88 \mathrm{c}$ \\
Cymbopogon citratus & $3,63 \mathrm{ab}$ \\
Eucalyptus citriodora & $4,00 \mathrm{a}$ \\
\hline
\end{tabular}

Médias seguidas por letras iguais na coluna não diferem entre si, pelo teste de verossimilhança ao nível de 5\%. 
Óleos essenciais presentes no flavedo de frutas cítricas em quantidades altas, podem exercer uma importante ação bio-reguladora nos esporos dos dois mais agressivos patógenos fúngicos de pós-colheita de frutas cítricas, Penicillium digitatum e Penicillium italicum. Como parte de uma pesquisa para investigar a ação biorreguladora de óleos essenciais de frutas cítricas, um estudo 'in vitro', a fim de determinar a atividade de componentes do óleo essencial de frutas cítricas (nonanal, citral, alfa-pineno, mirceno, alfa-felandreno e d-limoneno) foi avaliado para germinação de esporos de $P$. digitatum e $P$. italicum.Demonstrou-se ser o citral o composto mais ativo, em relação ao método e para as estirpes do fungo empregadas, que, na dose de $250 \mathrm{ppm}$, provou ser capaz de exercer uma ação fungistática em ambos patógenos (Caccioni et al., 1995). Os óleos essenciais de Citrus apresentam em sua composição as substâncias acetaldeído, ácido acético, alfa-felandreno, ácido benzóico, cariofileno, ácido cinâmico, cis-ocimeno, citral, citronelal, citronelol, cumarina, formaldeído, geraniol, linalol, mirceno, naringenina, nobiletina, p-cimeno, fenol, seselina, sinensetina, terpinoleno, timol e umbeliferona com atividade fungicida comprovada (USDA, 2003). Almada-Ruiz et al. (2003) verificaram que quatro flavonas isoladas do óleo essencial de laranja foram efetivos na inibição do crescimento micelial de Colletotrichum gloeosporioides, sendo que o aumento da concentração de flavonas foi diretamente proporcional à inibição do patógeno.

\section{CONCLUSÕES}

Ocorreu menor variação no $\mathrm{pH}$ nos frutos contidos no tratamento testemunha durante o experimento, sendo que esses frutos, ao final do mesmo, encontravam-se com os menores valores de $\mathrm{pH}$, diferindo dos valores obtidos dos frutos submetidos aos demais tratamentos.

Frutos submetidos ao tratamento com Citrus sinensis encontravam-se com o maior teor de acidez total titulável, seguindo-se os valores obtidos nos frutos tratados com Origanum majorana e Eucalyptus citriodora.

Os tratamentos com o óleo essencial de Citrus sinensis e com bicarbonato de sódio proporcionaram os maiores teores de sólidos solúveis totais.

A incidência de antracnose foi menor nos frutos submetidos ao tratamento com o óleo essencial de Citrus sinensis, comprovando-se "in vivo", a atividade fungitóxica do mesmo.

\section{REFERÊNCIAS BIBLIOGRÁFICAS}

ALMADA-RUIZ, E.; MARTÍNEZ-TÉLLEZ, M.A.; HERNÁNDEZ-ÁLAMOS, M.M.; VALLEJO, S.; PRIMO-
YÚFERA, E.; VARGAS-ARISPURO, I. Fungicidal potential of methoxylated flavones from citrus for in vitro control of Colletotrichum gloeosporioides, causal agent of anthracnose disease in tropical fruits. Pest

Management Science, Sussex, v.59, n.11, p.1245-1249, 2003.

ASSOCIATION OF OFFICIALANALYTICAL CHEMISTRY. Official methods of analysis. Washington, 1992. 1115p.

AWASTHI, R.K.; PANDEY, I.C. Physico-chemical composition and canning suitability of mango varieties. Indian Food Packer, New Delhi, v.34, n.3, p.60-63, May/ June 1980.

BENATO, E.A.; CIA, P.; SOUZA, N.L. Manejo de doenças de frutas pós-colheita. In: LUZ, W.C. Revisão

Anual de Patologia de Plantas, Passo Fundo, v.9, p.403440, 2001.

BERNIZ, P.J. Avaliação industrial de variedades de manga (Mangifera indica $L$.) para elaboração de nectar. 1984. 55p. Dissertação (Mestrado em Fisiologia Vegetal)-Universidade Federal de Viçosa, Viçosa, 1984.

CACCIONI, D.R.L.; DEANS, S.G.; RUBERTO, G. Inhibitory effect of citrus fruit essential oil components on Penicillium italicum and P. digitatum. Petria, v.5, n.2, p.177-182, 1995.

CARVALHO, C.R.L.; ROSSETTO, C.J.; DILZA MANTOVANI, M.B.; MORGANO, M.A.; CASTRO, J.V.; BORTOLETTO, N. Avaliação de cultivares de mangueiras selecionadas pelo Instituto Agronômico de Campinas comparada a outras de importância comercial. Revista Brasileira de Fruticultura, Jaboticabal, v.26, n.2, p.264-271, 2004.

CHITARRA, M.I.F.; CHITARRA, A.B. Pós-colheita de frutas e hortaliças: fisiologia e manuseio. Lavras: ESAL/ FAEPE, 2005. 735p.

CRUZ, M.E.S. Produtos alternativos no controle de doenças de pós-colheita de banana (Musa paradisiaca L.), maçã (Malus domestica Borkh) e laranja (Citrus sinensis (L.) Osbeck). 2003. 117p. Tese (Doutorado em Agronomia)-Universidade Estadual de Maringá, Maringá, 2003. 
CRUZ, M.E.S.; SCHWANESTRADA, K.R.F.; CLEMENTE, E.; CRUZ, M.J.S.; STANGARLIN, J.R. Extratos vegetais na conservação de frutos de banana e no controle de antracnose em pós-colheita. In: CONGRESSO BRASILEIRO DE OLERICULTURA, 45. 2005, Fortaleza. Revista Brasileira de Horticultura, Fortaleza, v.23, p.511, 2005a. Suplemento.

CRUZ, M.E.S.; SCHWANESTRADA, K.R.F.; CLEMENTE, E.; CRUZ, M.J.S.; STANGARLIN, J.R. Produtos naturais na conservação de maçãs e no controle de Penicillium expansum em pós-colheita. In: CONGRESSO BRASILEIRO DE OLERICULTURA, 45., 2005, Fortaleza. Revista Horticultura Brasileira, Fortaleza, v.23, p.511, 2005b. Suplemento.

FERREIRA, D.F. Análise estatística por meio do SISVAR (Sistema para Análise de Variância) para Windows versão 4.0. In: REUNIÃO ANUAL DA REGIÃO BRASILEIRA DA SOCIEDADE INTERNACIONAL DE BIOMETRIA, 45., 2000, São Carlos. Anais... São Carlos: UFSCar, 2000. p.255-258.

\section{INSTITUTO ADOLFO LUTZ. Normas analíticas do} Instituto Adolfo Lutz: métodos químicos e físicos para análises de alimentos. São Paulo: USP, 1985. v.1, 450p.

JACOMINO, A.P. Pós-colheita de frutas e hortaliças. Disponível em: 〈http://Www.agrorganica.com.brh 'pos_colheita.htmì. Acesso em: 17 jul. 2003.

KANESHIRO, M.A.B.; JERONIMO, E.M.; PARO, R.M.; MARQUES, M.O.; BERTINI, E.F.; TOSTES, D.R.D. Efeito de embalagem no armazenamento refrigerado de manga (Mangifera indica L.). In: SIMPÓSIO LATINO AMERICANO DE CIÊNCIAS DOS ALIMENTOS, 1995, Campinas. Anais... Campinas: FEA, 1995. p.94.

LEDERMAN, I.E. Determinação do ponto de colheita da manga cv. Tommy Atkins, para a região semi-árida de
Pernambuco. Revista Brasileira de Fruticultura, Cruz das Almas, v.20, n.2, p.145-151, 1998.

LEITE, L.A.S.; PESSOA, P.F.A.; ALBUQUERQUE, J.A.; SILVA, P.C.G. O agronegócios da manga no Nordeste. In: CASTRO A.M.G. Cadeias produtivas e sistemas naturais: prospecção tecnológica. Brasília: Embrapa-SPI, 1998. p.389-439.

LIMA, L.C. de O. Tecido esponjoso em manga

'Tommy Atkins': transformações químicas e bioquímicas no mesocarpo durante o armazenamento. 1997. 151p. Tese (Doutorado em Ciência dos Alimentos)-Universidade Federal de Lavras, Lavras, 1997.

MARTIM, N.S.P.P. Estudo das características de processamento da manga (Mangifera indica $\mathrm{L}$.) variedade Tommy Atkins desidratada. 2006. 76p. Dissertação (Mestrado em Tecnologia de Alimentos)Universidade Federal do Paraná, Curitiba, 2006.

MYERS, R.H.; MONTGOMERY, D.C.; VINING, G.G. Generalized linear models. New York: J.Wiley, 2002. $341 \mathrm{p}$.

SILVA, M.F.A.; MAIA, G.A.; HOLANDA, L.F.F.; MONTEIRO, J.C.S.; ORIA, H.F.; VASCONCELOS, P.M. Características físicas e químicas da manga. Ciência Agronômica, Fortaleza, v.17, n.1, p.73-80, jun. 1986.

SOARES JUNIOR, A.M.; MAIA, A.B.A.; NELSON, D.L. Estudo do efeito de algumas variáveis e fabricação no perfil texturométrico do doce de manga. Ciência e Tecnologia de Alimentos, Campinas, v.23, n.1, p.76-80, 2003.

UNITED STATE DEPARTMENT AGRICULTURE. National Genetic Resources Program. Phytochemical and ethnobotanical databases. Beltsville, 2003. 